\title{
Single Molecule Kinetics of ENTH Binding to Lipid Membranes
}

\author{
Sharon Rozovsky, ${ }^{1}$ Martin B. Forstner, ${ }^{2}$ Holger Sondermann ${ }^{3}$ and Jay T. Groves ${ }^{4,5}$
}

1. Department of Chemistry and Biochemistry, University of Delaware, Newark, Delaware.

2 Department of Physics, Syracuse University, Syracuse, New York.

3. College of Veterinary Medicine, Cornell University, Ithaca, New York.

4. Physical Biosciences and Materials Sciences Divisions, Lawrence Berkeley National Laboratory, Berkeley, California.

5 Howard Hughes Medical Institute, Department of Chemistry, University of California, Berkeley, California.

Transient recruitment of proteins to membranes is a fundamental mechanism by which cellular membranes govern biochemical reactivity. Such dynamic membrane recruitment of peripheral membrane proteins, i.e. proteins that shuttle between lipidic and cytoplasmic locations, exerts spatial and temporal control over proteins' localization and interactions. Following binding to the membrane, these proteins will encounter and interact with their partners. The Epsin N-terminal homology (ENTH) domain is a well-characterized protein domain that binds phosphatidylinositol (4,5)-bisphosphate (PtdIns $\left.(4,5) \mathrm{P}_{2}\right)$. It employs an amphipathic helix for membrane integration, formed upon binding of the target lipid. We measured the dissociation rate constant, diffusion rate and details of ENTH diffusion when bound to the membrane by detecting the residence time and diffusion of ENTH to planar supported fluid lipid bilayer (SLB). The direct visualization and quantification of interactions of single ENTH molecules with SLB was achieved using total internal reflection fluorescence microscopy (TIRFM) with a time resolution of $13 \mathrm{~ms}$. This time resolution allowed us detect to the kinetic behavior of ENTH with weakly charged membranes incorporating physiologically relevant concentrations of PtdIns $(4,5) \mathrm{P}_{2}$ despite the low effective binding affinity. Single fluorophore tracking analysis was used to recover the distributions of residence times and to measure ENTH dissociation rates as a function of membrane composition. The dissociation rate constant for $2,1,0.5$, and $0.1 \mathrm{~mol} \% \operatorname{PtdIns}(4,5) \mathrm{P}_{2}$ concentration was measured to be $4.5 \pm 1.6 \mathrm{~s}^{-1}, 6.8 \pm 2.5 \mathrm{~s}^{-1}, 12.5 \pm 2.5 \mathrm{~s}^{-1}$, and $14 \pm 6 \mathrm{~s}^{-1}$ respectively. The analysis of the step size distributions and the distribution of diffusion coefficients of individual proteins clearly show that the mode of transport of membrane associated ENTH is not simple Brownian diffusion described by one diffusion coefficient. Instead, we find that lateral diffusion of ENTH along the membrane is best described by 3 different diffusion processes. We hypothesize that they are associated with different modes of ENTH-membrane interactions [1]. 


\section{References:}

[1] Rozovsky, S., Forstner, M.B., Sondermann, H. and Groves, J. T. Binding kinetics of Epsin Nterminal Homology (ENTH) to lipid bilayers measured by single molecule total internal reflection microscopy. Journal of Physical Chemistry $116 \quad$ (17), 5122-5131 (2012). http://dx.doi.org/10.1021/jp210045r

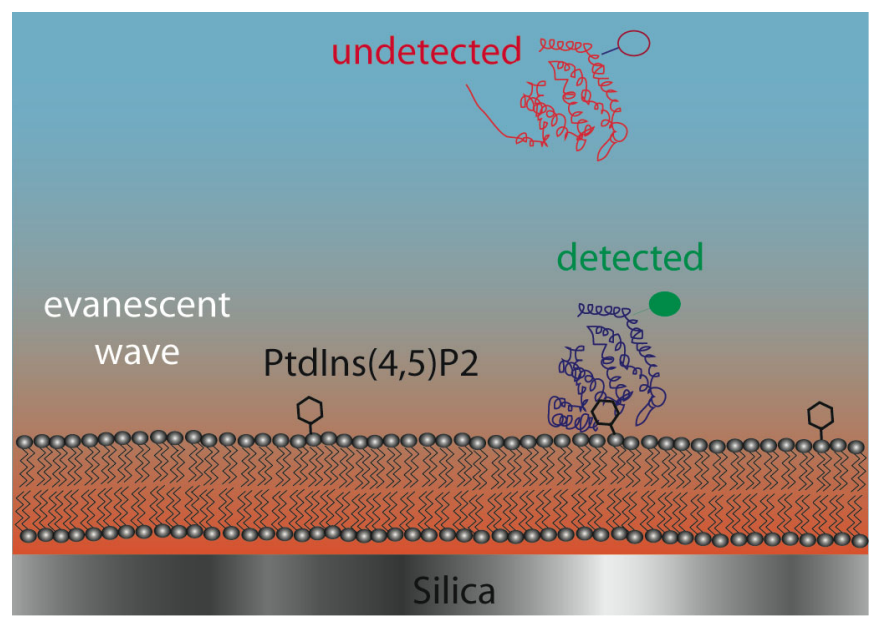

Figure 1. Experimental setup for detection of peripheral membrane proteins binding to glass supported lipid bilayer by total internal reflection fluorescence microscopy. Fluorescently labeled ENTH is allowed to equilibrate with a supported lipid bilayer containing its target lipid $\operatorname{PtdIns}(4,5) \mathrm{P}_{2}$ (black). The diffusion behavior and residence time of membrane bound ENTH is recorded using in the restricted illumination volume (orange). 